\title{
Quantitative contrast echocardiographic assessment of collateral derived myocardial perfusion during elective coronary angioplasty
}

\author{
S F de Marchi, M Schwerzmann, M Fleisch, M Billinger, B Meier, C Seiler
}

\begin{abstract}
Objective-To determine whether myocardial contrast echocardiography can be used to quantify collateral derived myocardial flow in humans.

Methods-In 25 patients undergoing coronary angioplasty, a collateral flow index (CFI) was determined using intracoronary wedge pressure distal to the stenosis to be dilated, with simultaneous mean aortic pressure measurements. During balloon occlusion, echo contrast was injected into both main coronary arteries simultaneously. Echocardiography of the collateral receiving myocardial area was performed. The time course of myocardial contrast enhancement in images acquired at end diastole was quantified by measuring pixel intensities (256 grey units) within a region of interest. Perfusion variables, such as background subtracted peak pixel intensity and contrast transit rate, were obtained from a fitted $\gamma$ variate curve.

Results-16 patients had a left anterior descending coronary artery stenosis, four had a left circumflex coronary artery stenosis, and five had a right coronary artery stenosis. The mean (SD) CFI was 19 (12)\% (range 0-47\%). Mean contrast transit rate was 11 (8) seconds. In 17 patients, a significant collateral contrast effect was observed (defined as peak pixel intensity more than the mean +2 SD of background). Peak pixel intensity was linearly related to CFI in patients with a significant contrast effect $(\mathrm{p}=0.002, r=0.69)$ as well as in all patients $(\mathrm{p}=0.0003, r=0.66)$.

Conclusions-Collateral derived perfusion of myocardial areas at risk can be demonstrated using intracoronary echo contrast injections. The peak echo contrast effect is directly related to the magnitude of collateral flow.
\end{abstract}

(Heart 2001;86:324-329)

Keywords: collateral circulation; quantitative myocardial contrast echocardiography; intracoronary pressure; myocardial perfusion

With increasing coronary obstruction, collateral vascular development variably occurs, allowing the myocardial areas jeopardised by ischaemia to receive blood from collateralsupplying coronary arteries. There is extensive evidence that after acute coronary occlusion, these bypass vessels exert a protective effect on the involved myocardium at risk for infarction. Collaterals are able to reduce the ischaemic bed size,${ }^{1}$ prevent ventricular aneurysm formation, preserve ventricular function, ${ }^{23}$ and improve survival. ${ }^{4}$ Experimental and clinical data suggest that collaterals also reduce ischaemia in the absence of coronary occlusion, particularly when myocardial oxygen demand is increased. ${ }^{56}$

In the past, the methods available to measure collateral flow, such as angiographic assessment, have been crude and have therefore contributed to much of the debate and confusion about the functional relevance of collaterals. On coronary angiography, collateral vessels are visible only if their diameter exceeds $100 \mu \mathrm{m}$, though they are often smaller than this. ${ }^{78}$ Intracoronary wedge pressure and Doppler flow velocity measurements have now been assessed to characterise collateral flow quantitatively in humans. ${ }^{9-11}$ With these techniques, it is possible to detect considerable collateral flow, even in patients without angiographic evidence of collaterals.
With the introduction of new generation echo contrast agents and advanced ultrasound techniques, myocardial contrast echocardiography has gained importance for the noninvasive assessment of blood flow at the level of myocardial perfusion. ${ }^{812}{ }^{13}$ Stable air or fluid filled microbubbles scattering ultrasound waves and thus producing intense echo enhancement are injected into the systemic or coronary circulation while cross sectional echocardiography is performed. As the rheology of the intravascular tracer is similar to that of red blood cells, ${ }^{14}$ regional myocardial perfusion can be quantified from the temporal and spatial distribution of myocardial contrast enhancement. ${ }^{15}$ In the past, applications of myocardial contrast echocardiography have focused mainly on the evaluation of myocardial viability and on rest versus hyperaemic flow, ${ }^{13}{ }^{16-18}$ but collateral derived myocardial perfusion has also been demonstrated qualitatively in some studies. ${ }^{19}{ }^{20}$ However, no study has been performed so far to assess collateral derived perfusion using myocardial contrast echocardiography in comparison with another quantitative method for determining collateral flow in humans.

Our aim in this study was to determine whether myocardial contrast echocardiography can be used for quantification of collateral derived myocardial flow in humans. 


\section{Methods}

STUDY POPULATION AND PROTOCOL

Twenty eight patients referred for elective coronary angiography at our laboratory were included in the study. Patients with acute coronary syndrome or previous $\mathrm{Q}$ wave infarction were excluded to avoid having myocardial areas with acute or previous microvascular damage. Of the 28 patients initially included, three were later withdrawn because no adequate echocardiographic image could be obtained.

Before percutaneous transluminal coronary angioplasty (PTCA), a bolus of echo contrast (Levovist, Schering, Berlin, Germany; see below) was injected into the patent coronary artery undergoing PTCA (that is, the collateral-receiving or ipsilateral coronary artery) and cross sectional echocardiography of the myocardial area at risk was performed, as described below. During balloon inflation, a bolus of echo contrast was injected simultaneously into both main coronary arteries by two operators, while transthoracic echocardiography of the same myocardial area was repeated. Distal intracoronary pressure, aortic pressure, and an intracoronary ECG were recorded continuously.

The study protocol was approved by the ethics committee of the University of Bern, Switzerland. Informed consent was obtained from the study participants.

PTCA AND PRESSURE MEASUREMENTS

Diagnostic coronary angiography was performed by the right femoral approach using 5 French Judkins catheters. For PTCA, 6 French guiding catheters were used. A second 5 French coronary catheter was placed in the ostium of the contralateral coronary artery for simultaneous bilateral echo contrast injections during vessel occlusion. Bilateral injections were used because the coronary artery supplying the collateral could not always be identified with absolute certainty. Coronary pressure measurements, including mean wedge pressure $\left(\mathrm{P}_{\text {occl }}\right)$, were performed using guide wires equipped with pressure sensors at their tip (PressureWire, RADI Medical, Uppsala, Sweden, or WaveWire, Endosonics, Rancho Cordova, California, USA). Mean aortic pressure $\left(\mathrm{P}_{\mathrm{ao}}\right)$ was measured through the ipsilateral guiding catheter. An intracoronary ECG obtained from the guide wire was recorded in addition to the standard limb leads. Collateral flow index (CFI) was calculated using the following formula: $\mathrm{CFI}=\left(\mathrm{P}_{\text {occl }}-\mathrm{CVP}\right) /\left(\mathrm{P}_{\mathrm{ao}}-\right.$ CVP), where central venous pressure (CVP) was assumed to be $5 \mathrm{~mm} \mathrm{Hg}$ in our study. ${ }^{9}$

MYOCARDIAL CONTRAST ECHOCARDIOGRAPHY

Transthoracic echocardiography was performed during the intervention in the catheterisation laboratory. All studies were performed on an Acuson Sequoia Doppler ultrasonography system (Acuson Inc, Mountain View, California, USA). Patients were placed in the supine position. An injection of $2 \mathrm{ml}$ of Levovist was given directly into the coronary arteries at a concentration of $300 \mu \mathrm{g} /$ $\mathrm{ml}$, followed by a flush of $10 \mathrm{ml} 0.9 \% \mathrm{NaCl}$ at a temperature of $37^{\circ} \mathrm{C}$. Echocardiography was performed using continuous harmonic imaging at a $3.5 \mathrm{MHz}$ transmitting and a $7 \mathrm{MHz}$ receiving frequency. The ultrasound machines were set at a high dynamic range $(70 \mathrm{~dB})$ and low baseline gain level in order to prevent signal compression at high intensities. Acoustic power was set at a mechanical index of 0.9-1.1, depending on echo quality. These settings were held constant throughout each study. Echocardiography of the myocardium of interest was performed during 30 seconds before and two minutes after contrast injection. The region of interest was chosen to include the vascular area undergoing PTCA - that is, the territory receiving the collaterals. The parasternal short axis view was generally preferred over apical views, in order to reduce overshadowing of the myocardium by contrast perfused areas.

OFF-LINE PIXEL INTENSITY MEASUREMENTS AND MYOCARDIAL PERFUSION INDICES

Images were recorded on S-VHS videotapes for off-line analysis. End diastolic images were digitised on a Tomtec personal computer using an ECG $\mathrm{R}$ wave trigger. A hand traced region of interest was placed within the myocardial area at risk, and pixel intensity (256 grey levels per pixel) was determined by videodensitometry to quantify the tissue microbubble concentration.

Perfusion dynamics of the echo contrast medium As wash in, increase to peak tissue concentration, and wash out of a contrast medium used in a two compartment model obeys an exponential law with respect to time, a $\gamma$ variate function $\left(y=b e^{-a x}\right)$ was fitted to the raw timeintensity data obtained from videodensitometry (fig 1). The contrast effect was defined as significant if the background-subtracted peak pixel intensity of the $\gamma$ curve exceeded the upper 2 SD limit of the background pixel intensity distribution acquired before contrast injection. Peak pixel intensity, the area under the raw time-intensity curve, the area under the fitted $\gamma$ variate curve, the time to wash in, and the transit rate were measured.

\section{STATISTICAL ANALYSIS}

Demographic, clinical, and echocardiographic data are expressed as mean (SD). For comparisons of continuous values between groups, unpaired Student's $t$ tests were performed. We used $\chi^{2}$ tests for comparison of categorical variables between groups. The $\gamma$ variate curve fittings were performed on Matlab 5.1 computer software according to the least squares fitting method. Relations between different variables were studied using linear or nonlinear regression analyses. For accuracy calculations, receiver-operator characteristics were analysed. A probability value of $\mathrm{p}<0.05$ was considered significant. 


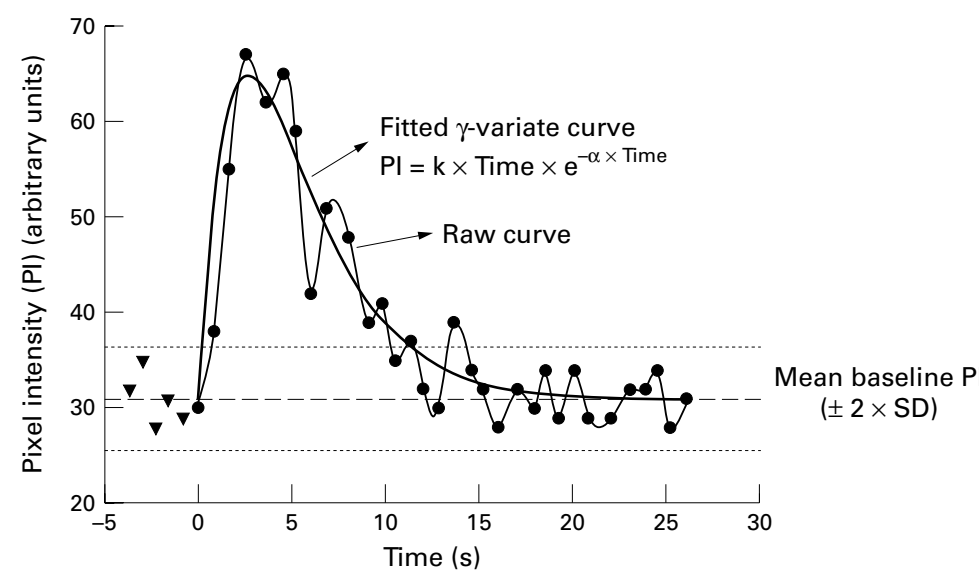

Figure 1 Illustration of the $\gamma$ variate curve fitting performed over the raw time-pixel intensity data. Raw data points of microbubble tissue concentration are given by triangles (background intensity of the myocardium before contrast injection) and by circles. Before the fitting procedure was started, the mean value and standard deviation at baseline and at the start of appearance of the contrast were determined. All peak values and area under the curve values obtained from the raw or $\gamma$ variate curve were calculated using background subtraction. The contrast effect was defined as significant if the background subtracted peak of the $\gamma$ curve (peak pixel intensity) exceeded the upper 2 SD limit (dotted line) of the background pixel intensity distribution.

\section{Results}

PATIENTS

The study patient characteristics are listed in table 1 . The mean (SD) CFI was 19 (12)\% (range $0-47 \%$ ).

REGIONS OF INTEREST

The size of the regions of interest was 641 (242) pixels. Of the 16 patients with left anterior descending coronary artery stenosis, the region of interest was placed within the anterior left ventricular wall in 11 patients and within the septal left ventricular wall in five. In all patients with left circumflex coronary artery or right coronary artery stenoses, the region of interest was placed within the posterolateral and inferior left ventricular wall.

TRANSIENT INTRACORONARY PRESSURE AND ECG CHANGES DURING CONTRAST INJECTION

During ipsilateral injection of contrast and saline before occlusion, a transient increase in mean intracoronary pressure from 77.0 (23.6) $\mathrm{mm} \mathrm{Hg}$ to 78.1 (24.3) $\mathrm{mm} \mathrm{Hg}$ was observed $(p=0.006)$. During occlusion, no injection induced changes of intracoronary wedge pressure could be measured because, owing to the ongoing vessel occlusion, the

Table 1 Characteristics of the study patients

\begin{tabular}{lc}
\hline $\mathrm{n}$ & 25 \\
Age (years) & $65(11)$ \\
Heart rate (beats/min) & $71(9)$ \\
Ejection fraction (\%) & $65(14)$ \\
1/2/3 vessel disease & $19 / 6 / 0$ \\
PTCA of: & \\
$\quad$ LAD stenosis & 16 \\
$\quad$ LCx stenosis & 4 \\
$\quad$ RCA stenosis & 5 \\
Size of region of interest (pixels) & $641(242)$ \\
Contrast transit rate (s) (ipsilateral & \\
$\quad$ injections before PTCA) & $14(9)$ \\
Contrast transit rate (s) (bilateral & \\
$\quad$ injections during PTCA) & $11(8)$ \\
\hline
\end{tabular}

Values are mean (SD) or $\mathrm{n}$.

LAD, left anterior descending coronary artery; LCx, left circumflex coronary artery; PTCA, percutaneous transluminal coronary angioplasty; RCA, right coronary artery. pressure signal was not stable at the time of contrast injection in most patients. In one patient, the ipsilateral injection was associated with mild transient ST segment elevation in the intracoronary ECG lasting 20 seconds, while bilateral contrast injection led to ST segment depression lasting 15 seconds. No chest pain or arrhythmias were observed.

DATA FROM PIXEL INTENSITY MEASUREMENTS

With ipsilateral injections before occlusion, a significant contrast effect was observed in 24 patients. Peak pixel intensity was 31 (13) units, the area under the fitted $\gamma$ variate curve was 1136 (882) units, the area under the raw timeintensity curve was 1186 (920) units/s, and the transit rate was 14 (9) s. With bilateral injections during vessel occlusion, a significant contrast effect was present in 17 patients (fig 1). Peak pixel intensity was 17 (12) units $(\mathrm{p}<0.0001 v$ ipsilateral injections during patency), the area under the fitted $\gamma$ variate curve was 577 (666) units/s $(\mathrm{p}<0.0001)$, the area under the raw time-intensity curve was 596 (719) units/s $(\mathrm{p}=0.007)$, and the transit rate was 11 (8) s (NS). CFI was 22 (13)\% in patients with, and 13 (9)\% in patients without, significant contrast effect during occlusion $(\mathrm{p}=0.07)$.

Over the entire range of intracoronary pressures before and during occlusion, there was a significant power law rise of peak pixel intensity with increasing pressure (fig 2). All peak pixel intensity data derived from bilateral injections during occlusion were located on the steep part of the regression curve, whereas for ipsilateral injections during vessel patency, the association between peak pixel intensity and intracoronary pressure was weak. The CFI was linearly related to peak pixel intensity in all patients $(\mathrm{p}<0.0003, r=0.66$; fig 3$)$. This correlation was closer when only patients with significant contrast effect during occlusion were included in the analysis ( $\mathrm{p}=0.002, r=0.69$ ). Furthermore, there was a significant positive linear relation between CFI and the area under

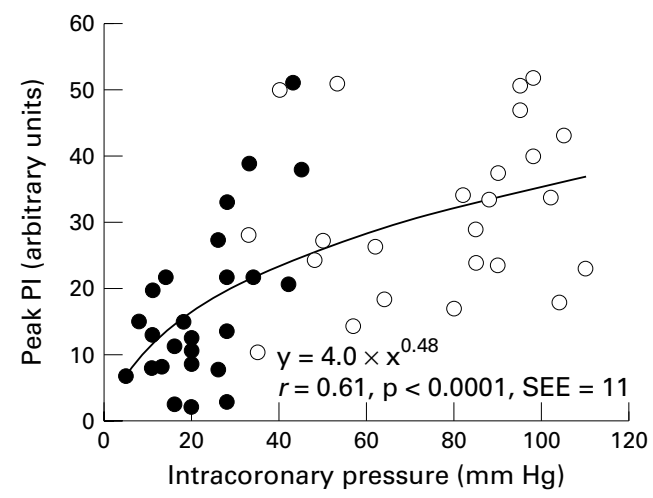

Figure 2 Peak pixel intensity as a function of intracoronary pressure measured after ipsilateral contrast injections during vessel patency (empty circles; $n=25$ ) and bilateral contrast injections during vessel occlusion (solid circles; $n=25$ ). There was a significant power-law relation between the two variables. The flattening of the curve may in part reflect signal compression at high intensities, whereas at lower intracoronary pressures (such as wedge pressure), a steep relation over the entire range of peak pixel intensities was present. Peak PI, peak pixel intensity; SEE, standard error of estimate. 


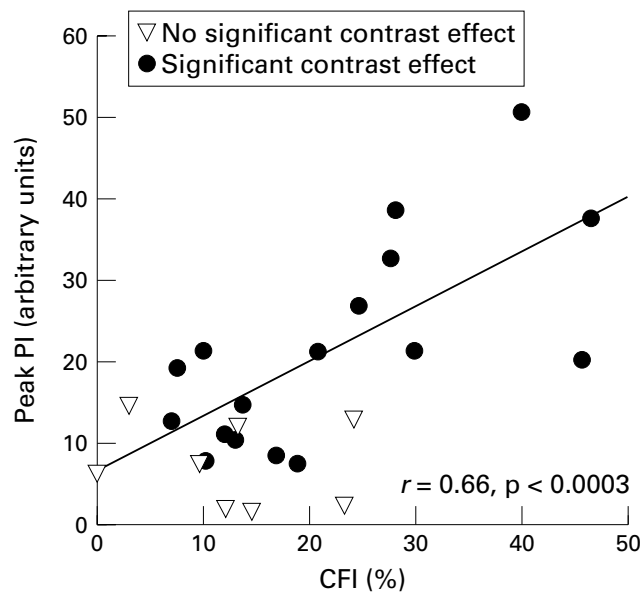

Figure 3 Peak pixel intensity as a function of collateral flow index calculated from intracoronary wedge pressure measurements. A significant positive linear relation was observed (all patients, $n=25$ ), particularly when only patients with significant contrast effect were analysed (solid circles, $r=0.69, p=0.002 ; n=18$ ). CFI, collateral flow index; peak PI, peak pixel intensity.

the fitted $\gamma$ variate curve $(\mathrm{p}=0.008, r=0.52)$, as well as the area under the raw time-intensity curve $(\mathrm{p}=0.008, r=0.52)$. The time to wash in was inversely related to CFI, but the correlation was weak ( $\mathrm{p}<0.05, r=-0.40)$. No correlation between $\mathrm{CFI}$ and transit rate was observed.

Examples of baseline and contrast enhanced echocardiography in a patient with poor collateral supply and a patient with abundant collateral supply are shown in fig 4 .

\section{ACCURACY CALCULATION}

A peak contrast effect of more than 20 pixel intensity units had a specificity of $86 \%$, a sensitivity of $82 \%$, a positive predictive value of $82 \%$, and a negative predictive value of $86 \%$ for the detection of a CFI of $\geqslant 20 \%$.

\section{Discussion}

This study in patients without previous myocardial infarction shows that coronary collateral flow can be quantified using contrast echocardiography. The peak contrast effect within the collateral receiving myocardial area, as well as the area under the time-intensity curve and the time to wash in of the contrast medium, correlated with simultaneously obtained, pressure derived collateral flow index measurements. Peak contrast pixel intensity
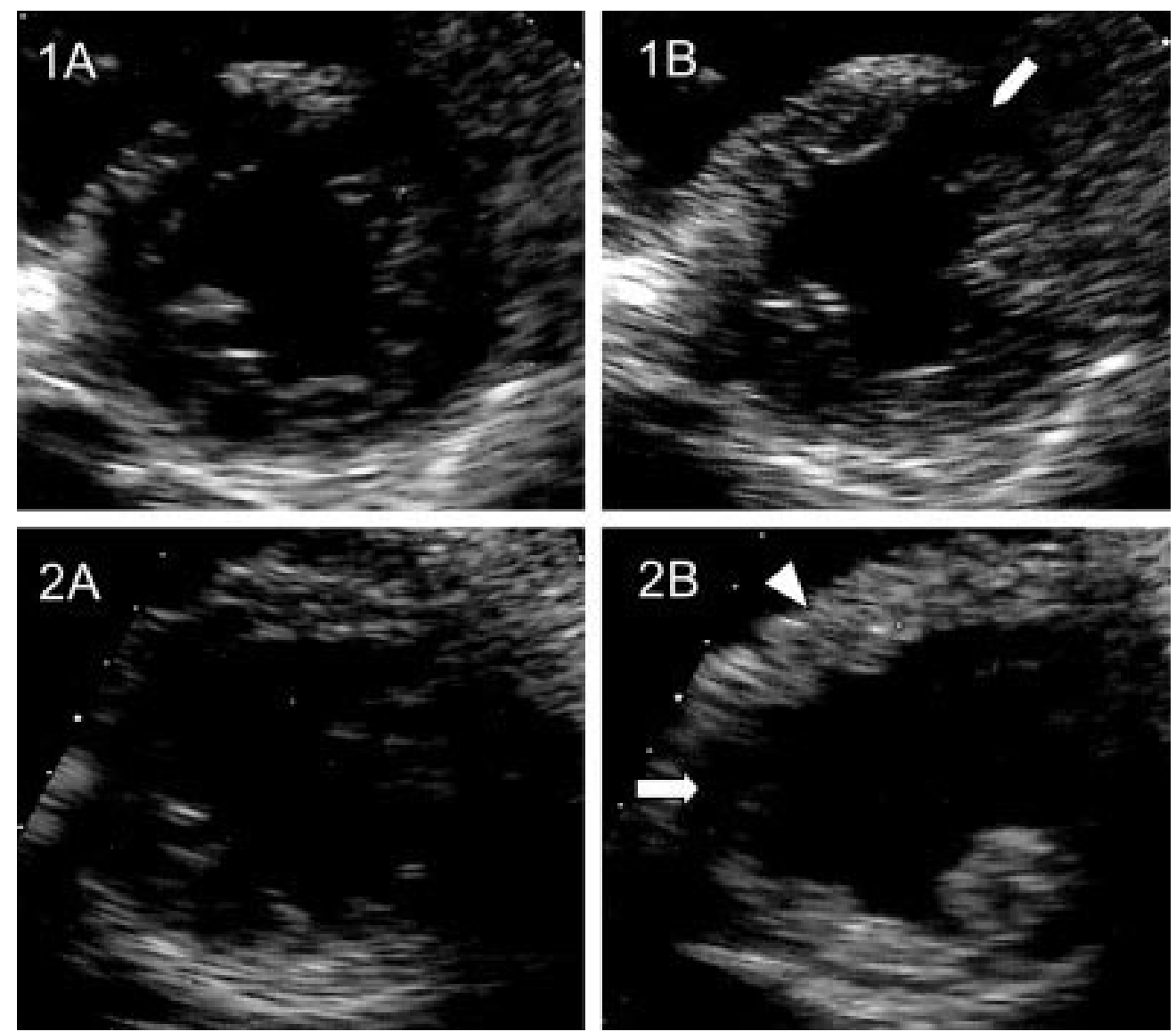

Figure 4 Examples of baseline $(A)$ and contrast enhanced echocardiography $(B)$ in a patient with poor collateral supply $(1 \mathrm{~A}, 1 \mathrm{~B} ; \mathrm{CFI}=9.7 \%)$ and one with abundant collateral supply $(2 \mathrm{~A}, 2 \mathrm{~B} ; \mathrm{CFI}=40.0 \%)$. Bilateral intracoronary injections of contrast were performed during coronary occlusion. Patient 1 underwent dilatation of a left anterior descending coronary artery stenosis distal to the septal branches and first diagonal branch. The arrow in panel $1 B$ indicates the myocardial region distal to the occluded coronary artery, where a consistent lack of contrast enhancement was observed. Patient 2 underwent dilatation of the proximal left anterior descending coronary artery. After bilateral intracoronary contrast injection during vessel occlusion, the septum and left ventricular anterior wall clearly showed contrast enhancement, indicating abundant collateral flow (arrowhead). Arrow: inferoseptal myocardial area of contrast overshadowing. CFI, collateral flow index. 
appears to be the most accurate of the variables obtained to measure collateral flow.

DATA FROM PREVIOUS STUDIES

Several contrast echocardiographic studies have been performed for qualitative detection of collateral derived myocardial perfusion in the clinical setting. Spotnitz et al carried out intraoperative studies in six patients with severe left coronary artery disease undergoing coronary artery bypass grafting. ${ }^{20}$ They showed that the collateral derived peak contrast effect within anterior regions improved after placement of a graft to the posterior descending coronary artery, even in patients without demonstrable collateral vessels at preoperative coronary angiography. Lim et al found a relation between angiographic collateral grade and collateral derived peak contrast effect after contralateral intracoronary contrast injection in 29 patients with total coronary occlusion and healed myocardial infarction. ${ }^{19}$ In an experimental study using the radiolabelled microsphere technique as a reference for the assessment of collateral flow, Cheirif et al showed that contrast echocardiography was capable of estimating the magnitude of coronary collateral flow and could quantitate the changes induced by occlusion and reperfusion. ${ }^{21}$ Grill et al studied 24 patients without coronary occlusion and found that contrast echocardiography using subsequent ipsilateral and contralateral intracoronary contrast injections was capable of mapping the collateral receiving territory and detecting changes in collateral supply induced by angioplasty. ${ }^{22}$ In 21 patients with previous myocardial infarction, left ventricular wall motion abnormality, and a chronically occluded coronary artery, Vernon et al showed a positive correlation between regional wall motion score and collateral receiving area size determined by contrast echocardiography after intracoronary contrast injections, indicating that collateral derived perfusion is associated with preserved regional function in patients with total coronary occlusion. ${ }^{23}$ Interestingly, no correlation between wall motion and angiographic collateral grade was found in that study, proving the inability of angiography to provide sufficient relevant functional information on coronary collaterals. Similar results were obtained by Sabia et al, ${ }^{8}$ who concluded that contrast echocardiography may be particularly suitable for assessing collaterals in patients undergoing cardiac catheterisation.

The studies on myocardial contrast echocardiography and collaterals published so far have not fulfilled the mandatory requirements for evaluating this technique in the clinical setting. Thus total coronary occlusion (natural or during PTCA) - which is required to avoid concomitant antegrade flow of contrast-was not present in all the studies. Patients with known damage to the myocardial microcirculation were often included. Furthermore, inadequate reference methods were commonly used-for example angiographic collateral grading systems.
COLLATERALS AND PEAK CONTRAST EFFECT Our study was performed in patients without previous myocardial infarction who were undergoing coronary angiography for suspected coronary artery disease. Bilateral intracoronary echo contrast injections during vessel occlusion were performed in order to allow all coronary collaterals to contribute to the opacification of the collateral receiving bed. The positive correlation between peak contrast effect and the magnitude of collateral flow may reflect two basic mechanisms. First, with increasing collateral supply, the collateral receiving bed size increases. Because the region of interest was placed in the centre of the area at risk, high peak pixel intensity may reflect an extension of collateral derived perfusion from the margin of the contralateral region to the centre of the ipsilateral area at risk. However, as no changes in area at risk between ipsilateral and contralateral injection were determined, this has to be remain speculative. Second, with increasing collateral supply, intramyocardial blood volume increases, thus producing increasingly homogeneous and less scattered opacification of the collateral receiving myocardium. High peak pixel intensity may therefore reflect an increase in contrast concentration within the region of interest, independent of variations in the collateral receiving area size.

Nevertheless, it is clear that there is a remarkable scatter in the correlation between peak pixel intensity and CFI (fig 3). Several technical factors-such as difficult echocardiographic imaging conditions during coronary interventions with the patient supine, as well as non-standardised echo windows - are crucial to the results and may have contributed to artificial contrast enhancement in some patients. However, regional contrast heterogeneity is reported to be common, even in patients without coronary artery disease, resulting in frequent false positive prediction of perfusion defects. ${ }^{24}$ In the present study, the correlation between peak pixel intensity or the area under the time-intensity curve and CFI can probably not be expected to be closer, as experimental data on collateral flow published by Cheirif et al showed a correlation coefficient of 0.72 for the comparison of the radiolabelled microsphere method with contrast echocardiography in an open chest dog model. ${ }^{21}$ Diagnostic accuracy calculations in our study, however, showed promising results for distinguishing between high and low collateral flow.

\section{COLLATERALS AND CONTRAST TRANSIT RATE}

Previous studies by Kaul et al have shown that the contrast transit rate reflects myocardial blood flow. ${ }^{25}$ In our study, however, no correlation was found between CFI and contrast transit rate. The time course of microbubble transit through the myocardial microvasculature largely depends on the contrast inputconcentration curve. A $\gamma$ variate curve fitting procedure is most appropriate when the complete contrast bolus enters a dilution compartment very rapidly, such as in the microcirculation of collateral supplied myocardium. In our study, no mechanically assisted injections 
were performed. Thus the input curve may have varied considerably between patients, depending on concomitant stenoses within the collateral supplying arteries, catheter size, and other factors. Furthermore, it must be considered that coronary collateral vessels may produce an additional dilution of contrast, further widening the input curve and affecting the transit rate calculation. In patients with poor collateral supply, the width of the fitted $\gamma$ variate curve varies greatly because no curve fitting is appropriate in flat time-intensity curves. However, the peak of the $\gamma$ variate curve remains consistently low in these patients, regardless of the curve width found in the fitting operation.

LIMITATIONS OF THE STUDY

Aside from the limitations alluded to above, we need to mention that the contrast injection durations may have been longer than in previous studies, causing a longer period of transition of the contrast through the microcirculation. The calculated transit rate is therefore somewhat longer than expected. Occlusion of the microcirculation caused by inappropriate microbubble size is, however, improbable because signs of ischaemia in the intracoronary ECG were absent during all but two injections in two patients. In these two patients, large air bubbles entrapped invisibly within the white contrast material were probably injected into the coronary artery, leading to reversible signs of ischaemia in both cases and transient left ventricular systolic dysfunction on echocardiography in one patient. During regular diagnostic coronary angiography, accidental injection of air into the coronary artery occurs infrequently. However, this seldom leads to more than transient ECG signs of myocardial ischaemia and short lasting chest pain.

To ensure optimal visibility of the myocardial area of interest, the acoustic window was not standardised in this study. This may have produced an additional variability in pixel intensity measurements compared with studies in which only parasternal short axis views were used.

Patients were not screened for myocardial viability using scintigraphic scans as a part of the study protocol. Therefore, pre-existing perfusion defects within the collateral receiving bed cannot entirely be excluded.

CONCLUSIONS

Myocardial contrast echocardiography provides important information on collateral flow during coronary angioplasty. In patients without previous myocardial infarction, the peak collateral derived contrast effect is positively correlated with collateral flow as assessed by intracoronary wedge pressure measurements. Our results may be important in developing non-invasive methods for assessing collateral derived myocardial perfusion.
This study was supported by a grant of the Swiss National Science Foundation, grant No 3200-058945.99 (to CS).

1 Habib GB, Heibig J, Forman SA, et al. Influence of coronary collateral vessels on myocardial infarct size in humans. Results of phase I thrombolysis in myocardial infarction Results of phase I thrombolysis in myocardial infarction (TIMI) trial.

2 Hirai T, Fujita $M$, Nakajima $H$, et al. Importance of collateral circulation for prevention of left ventricular aneurysm formation in acute myocardial infarction. Circulation 1989; 79:791-96.

3 Sabia PJ, Powers ER, Ragosta M, et al. An association between collateral blood flow and myocardial viability in
patients with recent myocardial infarction. $N$ Engl $\mathcal{F}$ Med 1992;327:1825-31.

4 Hansen J. Coronary collateral circulation: clinical significance on survival in patients with coronary artery occlusion. Am Heart f 1989;117:290-5.

5 Senti S, Fleisch M, Billinger M, et al. Long-term physical exercise and quantitatively assessed human coronary collateral circulation. $\mathcal{F}$ Am Coll Cardiol 1998;32:49-56.

6 Billinger M, Kloos P, Fleisch M, et al. Coronary collateral flow and its protective potential against future myocardial infarctions. Eur Heart f 1999;20:646.

7 Gensini GG, Bruto da Costa BC. The coronary collateral circulation in living man. Am f Cardiol 1969;24:393-400.

8 Sabia PJ, Powers ER, Jayaweera AR, et al. Functional significance of collateral blood flow in patients with recent acute myocardial infarction. A study using myocardial contrast echocardiography. Circulation 1992;85:2080-9.

9 Seiler C, Fleisch M, Garachemani A, et al. Coronary collateral quantitation in patients with coronary artery disease using intravascular flow velocity or pressure measurements. using intravascular flow velocity or pres

10 Seiler C, Fleisch M, de Marchi S, et al. Functional assessment of collaterals in the human coronary circulation. Semin Intervent Cardiol 1998;3:13-20.

11 Meier B, Luethy P, Finci L, et al. Coronary wedge pressure in relation to spontaneously visible and recruitable collaterals. Circulation 1987;75:906-13.

12 Kaul S. Clinical applications of myocardial contrast echocardiography. Am $\mathcal{F}$ Cardiol 1992;69:46-55H

13 Kaul S. Myocardial contrast echocardiography: 15 years of research and development. Circulation 1997;96:3745-60.

14 Jayaweera AR, Edwards N, Glasheen WP, et al. In vivo myocardial kinetics of air-filled albumin microbubbles during myocardial contrast echocardiography. Comparison with radiolabeled red blood cells. Circ Res 1994;74:1157-65.

15 Villanueva FS, Kaul S. Assessment of myocardial perfusion in coronary artery disease using myocardial contrast echocardiography. Coron Artery Dis 1995;6:18-28.

16 Cheirif J, Zoghbi WA, Raizner AE, et al. Assessment of myocardial perfusion in humans by contrast echocardiography. I. Evaluation of regional coronary reserve by peak contrast intensity. F Am Coll Cardiol 1988;11:735-43.

17 Keller MW, Glasheen W, Smucker ML, et al. Myocardial contrast echocardiography in humans. II. Assessment of coronary blood flow reserve. 7 Am Coll Cardiol 1988;12: 925-34.

18 Agati L, Voci P, Bilotta F, et al. Dipyridamole myocardial contrast echocardiography in patients with single-vessel coronary artery disease: perfusion, anatomic, and funccoronary artery disease: perfusion, anatomic,
tional correlates. Am Heart f 1994;128:28-35.

19 Lim YJ, Nanto S, Masuyama T, et al. Coronary collaterals assessed with myocardial contrast echocardiography in
healed myocardial infarction. Am $\mathcal{F}$ Cardiol 1990;66:55661 .

20 Spotnitz WD, Matthew TL, Keller MW, et al. Intraoperative demonstration of coronary collateral flow using myocardial contrast two-dimensional echocardiography. Am $\mathcal{F}$ Cardiol 1990;65:1259-61

21 Cheirif J, Narkiewicz-Jodko JB, Hawkins HK, et al. Myocardial contrast echocardiography: relation of collateral perfusion to extent of injury and severity of contractile dysfunction in a canine model of coronary thrombosis and reperfusion. $\mathcal{F} \mathrm{Am}$ Coll Cardiol 1995;26:537-46.

22 Grill HP, Brinker JA, Taube JC, et al. Contrast echocardiographic mapping of collateralized myocardium in humans
before and after coronary angioplasty. $\mathcal{F} \mathrm{Am}$ Coll Cardiol 1990;16:1594-600.

23 Vernon SM, Camarano G, Kaul S, et al. Myocardial contrast echocardiography demonstrates that collateral flow can preserve myocardial function beyond a chronically occluded coronary artery. Am f Cardiol 1996;78:958-60.

24 Bach DS, Muller DW, Cheirif J, et al. Regional heterogeneity on myocardial contrast echocardiography without severe obstructive coronary artery disease. Am f Cardiol 1995;75: 982-6.

25 Kaul S, Kelly P, Oliner JD, et al. Assessment of regional myocardial blood flow with myocardial contrast twodimensional echocardiography. 7 Am Coll Cardiol 1989;13: $468-82$. 